\title{
Uso do método RGT (Repertory Grid Technique) como ferramenta para análise de produto
}

\author{
Usage of the RGT (Repertory Grid Technique) method as a product analysis \\ tool
}

\author{
DEMAISON, ANDRÉ \\ Doutorando em Design pela Universidade Estadual Paulista (UNESP) \\ demaison@gmail.com \\ ANDRADE, SARAH \\ Graduanda em Design pela Universidade Federal do Maranhão (UFMA) \\ sarahorandrade@gmail.com
}

FRANCO, MARISA

Graduanda em Informática Biomédica (UFPR)

marisafranco@gmail.com

PASCHOARELLI, LUIS CARLOS

Professor Titular da Universidade Estadual Paulista (UNESP)

luis.paschoarelli@unesp.br

\section{RESUMO}

Este estudo objetivou abordar o uso do método RGT (Repertory Grid Technique) (KELLY, 1955) como ferramenta para análise de produtos no campo do Design. Por se basear na teoria dos construtos pessoais, do campo da psicologia, o RGT permite ao pesquisador e avaliador "observar" os produtos por meio da ótica de cada participante. Um teste laboratorial foi conduzido, analisando escovas de dentes: participaram onze estudantes, sendo seis da área do design e cinco de outras áreas. Com os resultados, foi possível observar as implicações do método e suas peculiaridades para levantamento de dados projetuais relevantes, bem como a viabilidade na obtenção de dados qualitativos e quantitativos. As escalas de avaliação existentes no método ainda trazem luz a diversas discussões e interpretações possíveis no campo do design e da ergonomia.

RGT, produto, design, metodologia. 


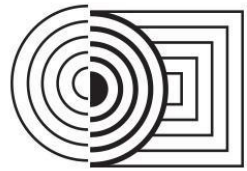

\section{$17^{\circ}$ ERGODESIGN \& USIHC 2019 \\ PUC-Rio, 11 a 13 de dezembro \\ Rio de Janeiro, RJ, Brasil}

$17^{\circ}$ Ergodesign - Congresso Internacional de Ergonomia e Usabilidade de Interfaces Humano Tecnológica: Produto, Informações Ambientes Construídos e Transporte

$17^{\circ}$ USIHC - Congresso Internacional de Ergonomia e Usabilidade de Interfaces Humano Computador

\begin{abstract}
This study aimed to address the use of the RGT (Repertory Grid Technique) method (KELLY, 1955) as a tool for product analysis in the Design field. Because it is based on the theory of personal constructs in the psychology field, the RGT allows the researcher and evaluator to "observe" the products through the perspective of each participant. A laboratory test analyzing toothbrushes was conducted: eleven students participated, being six from the design field and five from other fields of study. With the results, it was possible to observe the implications of the method and its peculiarities to survey relevant design data, as well as the feasibility in obtaining qualitative and quantitative data. The method's existing rating scales still shed light on several possible discussions and interpretations in the field of design and ergonomics.
\end{abstract}

$R G T$, product, design, methodology.

\title{
1. INTRODUÇÃO
}

A utilização do método RGT (Repertory Grid Technique) em análises de produtos, eventos e demais fenômenos ligados ao design tem sido assertiva, conforme observado nos estudos de Fallman (2006), Hogan e Horneker (2013) e Tomico et al. (2009). Segundo Gkouskos, Normark e Lundgren (2014), "o RGT origina-se da teoria do construto pessoal (proposta por George Kelly, em 1955), que é uma teoria da cognição humana. A principal suposição é que a compreensão individual do mundo consiste em dimensões de semelhanças e diferenças chamadas construtos pessoais". Tal teoria se baseia na visão peculiar de cada indivíduo acerca do fenômeno analisado, buscando em seu próprio repertório uma propriedade que o permita fazer uma avaliação. É possível encontrar a aplicação do RGT para avaliação da imagem de shopping centers (HERNANDEZ, 2005); para o desenvolvimento da personalidade de uma marca de luxo (HEINE, 2009); requisitos para projetos de produto (HASSENZAHL; WESSLER, 2000); e até mesmo para inferir as possíveis necessidades de motoristas/usuários de carros em futuros utópicos ou distópicos (GKOUSKOS; NORMARK; LUNDGREN, 2014).

A versatilidade e a quantidade de dados possíveis de se extrair do RGT fazem do método uma escolha bastante adequada para levantamento de informações relevantes para o design (HASSENZAHL; WESSLER, 2000). Para Fransella, Bell e Bannister (2004), ao desenvolver o método, George Kelly procurou trazer a matemática, ciência pela qual nutria imensa admiração (conforme Hinkle, 1970) para sua teoria psicológica. Segundo os autores, "ele escolheu fazer isso criando a grade (grid) do repertório. Ele viu a grade (grid) como nada mais e nada menos que outra maneira de afirmar sua teoria das construções pessoais. Não é um 'complemento'. É a teoria do construto pessoal em ação" (p. 1).

O presente estudo teve como objetivo observar o comportamento do RGT na análise de escovas de dente, por alunos de graduação na área do Design e de outras áreas. Visou extrair o máximo de informações possíveis em relação aos objetos analisado, permitindo a compreensão do método para aplicações em estudos da área do Design e Ergonomia. A aplicação do RGT foi realizada por uma equipe de alunos e professores do Fabrique Design Núcleo de Prototipagem do curso de Design da Universidade Federal do Maranhão e do Programa de Pós-graduação em Design da Universidade Estadual Paulista. 


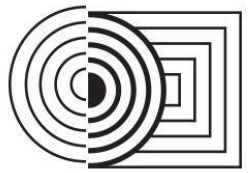

\section{$17^{\circ}$ ERGODESIGN \& USIHC 2019 \\ PUC-Rio, 11 a 13 de dezembro \\ Rio de Janeiro, RJ, Brasil}

$17^{\circ}$ Ergodesign - Congresso Internacional de Ergonomia e Usabilidade de Interfaces Humano Tecnológica: Produto, Informações Ambientes Construídos e Transporte

$17^{\circ}$ USIHC - Congresso Internacional de Ergonomia e Usabilidade de

Interfaces Humano Computador

\section{O RGT COMO FERRAMENTA DE AVALIAÇÃO: PROPRIEDADES E POSSIBILIDADES}

Conforme observado por Hassenzahl e Wessler (2000), "o design de um artefato (por exemplo, sistema de software ou um simples eletrodoméstico) requer várias decisões. Durante o processo de design, as 'boas ideias' precisam ser separadas das 'más ideias'. Para isso, as percepções e avaliações dos usuários são de grande valor". Para Tomico et al. (2009), "no campo $\mathrm{HCl}$, o RGT é usado para explorar as maneiras pelas quais os indivíduos percebem e diferenciam os produtos". Segundo Heine (2009, p.28, tradução nossa), "o objetivo do RGT é descobrir o repertório de construções pessoais. O desafio é que eles costumam ser difíceis de expressar em distinções verbais, porque muitos deles são subconscientes". Nos termos de Kelly (conforme apresentado por FRANSELLA; BELL; BANNISTER, 2004), todos "somos 'cientistas' que derivam hipóteses (têm expectativas) de nossas teorias (nossa interpretação pessoal). Submetemos essas hipóteses a testes experimentais (apostamos nelas comportamentalmente e assumimos riscos ativos em termos delas)".

Hernandez (2005) afirma que o "RGT compreende dois aspectos fundamentais: a forma como os dados são coletados e a forma como esses dados são analisados". Basicamente, cabe ao pesquisar provocar o participante, durante a aplicação do teste, a explorar seu repertório pessoal e atribuir ao objeto de estudo características ou propriedades, também chamado por alguns autores de "construtos", apesar das nomenclaturas parecerem se misturar em alguns momentos. No estudo de Gkouskos, Normark e Lundgren (2014), por exemplo, em determinados pontos é atribuído o nome de construto à característica dada pelo participante, em outros os autores colocam que o construto é, na verdade, a classificação bipolar (propriedade e seu oposto) colocada pelo sujeito. Conforme Fransella, Bell e Bannister (2004), "em todas as suas definições, Kelly mantém a noção essencial de que os construtos são bipolares. Seu argumento é que nunca afirmamos nada sem negar algo simultaneamente. Isso torna a noção de um construto bem diferente da noção de um conceito". Fernandes (2001) coloca que:

Um construto é a unidade básica de construção de significado, consistindo essencialmente numa discriminação entre elementos, sendo que a construção de uma experiência ou acontecimento tem subjacente uma afirmação e uma negação simultâneas.

(FERNANDES, 2001, p. 82)

Partindo do princípio de que, na teoria dos construtos ,"cada pessoa é capaz de formar um sistema de construtos na interpretação dos acontecimentos diários" (Hernandez, 2005), é possível assumir que "os construtos pessoais (e os tópicos subjacentes) gerados como uma reação a um conjunto de artefatos marcam o 'espaço de design'11 dos artefatos da perspectiva do usuário e que essas informações podem ser úteis para separar ideias valiosas das não tão valiosas" (Hassenzahl e Wessler 2000). Outro aspecto relevante do RGT é a possibilidade de se analisar questões relativas à cultura dos participantes. Considerando que o método traz ao pesquisador a visão a partir da ótica do entrevistado, torna-se possível separar as opiniões pessoais (ou construtos) por diversas categorias - incluindo aí informações que digam respeito ao repertório cultural do indivíduo. Tomico et al. (2009) apresentam um estudo no qual utilizam o RGT como método de estudo de diferenças culturais. Para os autores, "com o surgimento do

\footnotetext{
${ }^{1}$ Informações relevantes pro design, conforme o autor (tradução nossa).
} 


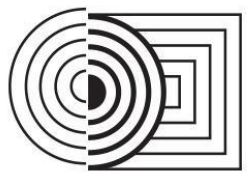

\title{
$17^{\circ}$ ERGODESIGN \& USIHC 2019 \\ PUC-Rio, 11 a 13 de dezembro \\ Rio de Janeiro, RJ, Brasil
}

$17^{\circ}$ Ergodesign - Congresso Internacional de Ergonomia e Usabilidade de Interfaces Humano Tecnológica: Produto, Informações Ambientes Construídos e Transporte

$17^{\circ}$ USIHC - Congresso Internacional de Ergonomia e Usabilidade de Interfaces Humano Computador

mercado global, as diferenças interculturais estão se tornando cada vez mais reconhecidas como um fator-chave na adoção bem-sucedida de novos produtos".

Hogan e Horneker (2013, p. 2, tradução nossa) explicam ainda que, "embora a técnica RepGrid $^{2}$ tenha sido inicialmente concebida como um método para uso em psicologia clínica, ao longo do tempo foi utilizada em diversos campos de pesquisa, como educação, política, marketing e sistemas de informação. Gkouskos, Normark e Lundgren (2014) explicam que o RGT se baseia no fato de que cada pessoa tem sua própria opinião - ou conjunto de noções que sejam relevantes a algo (objeto, serviço, fenômeno). Segundo eles:

\begin{abstract}
Por exemplo, quando se trata de uma máquina de lavar louça, Robin pode ser muito interessado em aspectos relacionados ao ruído e à economia, enquanto Kim está mais interessada em aspectos ambientais. Alguns construtos são fáceis de prever e encontrar, enquanto outros podem ser mais sutis, por exemplo, a rapidez com que a máquina funciona ou se é auto-limpante (GKOUSKOS; NORMARK; LUNDGREN, 2014, p. 62, tradução nossa).
\end{abstract}

Tal colocação explica a capacidade avaliativa do RGT, seja de maneira qualitativa ou quantitativa. Tomico et al. (2009) colocam que "devido à sua natureza qualitativa-quantitativa híbrida, (o RGT) permite um amplo tipo de análises estatísticas". O método ainda se mostrou versátil a ponto de possibilitar mesclas com outras técnicas, como o Grupo de Foco (Focus Group), como apresentado por Hogan e Horneker (2013). Conforme afirmam Fallman e Waterworth (2006), o RGT também mostrou "ser uma técnica útil para extrair significado em vários domínios diferentes, por exemplo, em gestão organizacional, educação, psicologia clínica e, particularmente, no desenvolvimento de sistemas baseados em conhecimento".

Hernandez (2005) explica as três maneiras propostas por George Kelly para se coletar as informações por parte dos participantes. Conforme o autor, são utilizados os métodos de (i) triadding (ou tríade), que consiste em apresentar três elementos ao participante para que se obtenha uma propriedade compartilhada por dois desses três elementos, processo repetido até que todas as tríades se esgotem ou que não se consiga mais elaborar construtos; (ii) laddering (ou escada), que basicamente consiste em provocar no participante respostas sobre o porquê de cada construto ser importante para ele; e (iii) pyramiding, quando se tenta obter do participante dados sobre a definição de cada polo do construto. Conforme observado nos trabalhos de Gkouskos, Normark e Lundgren (2014), Gkouskos e Normark (2012), Tomico et al. (2009), Karapanos e Martens (2008) e Falmann (2006), o método triádico é utilizado constantemente com resultados positivos no tocante a análises relativas ao design de produtos.

\section{MATERIAL E MÉTODOS}

O presente estudo caracteriza-se como transversal e exploratório. Foi desenvolvido em situação experimental e laboratorial. Foi desenvolvido a partir de um teste com o RGT para avaliação de escovas de dentes (Figura 01), com uma amostragem de participantes não-estatística, a fim de se obter dados para discussão e avaliação da viabilidade do método de maneira prática. Para o teste, o protocolo estabelecido prevê que o usuário/participante tenha contato com uma variedade de produtos e, a partir do seu entendimento (cognição), atribua construtos relacionados a eles. Posteriormente à atribuição dos construtos, o participante foi convidado a

\footnotetext{
${ }^{2}$ abreviação utilizada pelos autores para "Repertory Grid".
} 
$17^{\circ}$ ERGODESIGN \& USIHC 2019

PUC-Rio, 11 a 13 de dezembro

Rio de Janeiro, RJ, Brasil $17^{\circ}$ Ergodesign - Congresso Internacional de Ergonomia e Usabilidade de Interfaces Humano Tecnológica: Produto, Informações Ambientes Construídos e Transporte

$17^{\circ}$ USIHC - Congresso Internacional de Ergonomia e Usabilidade de Interfaces Humano Computador

avaliar o conjunto de produtos em uma escala de avaliação existente na tabela a partir das características bipolares dos construtos. Tais construtos são, então, categorizados em uma tabela juntos a um sistema de classificação, que permite ao pesquisador analisar o fenômeno estudado. Gkouskos, Normark e Lundgren (2014) afirmam que o RGT pode gerar dados "com diferentes níveis de envolvimento do pesquisador, variando do pesquisador decidir quais elementos para colocar no método (o que comparar)", permitindo assim flexibilidade na análise por parte da equipe pesquisadora.

Figura 01. Escovas de dentes utilizadas nos testes. (Fonte: os autores)

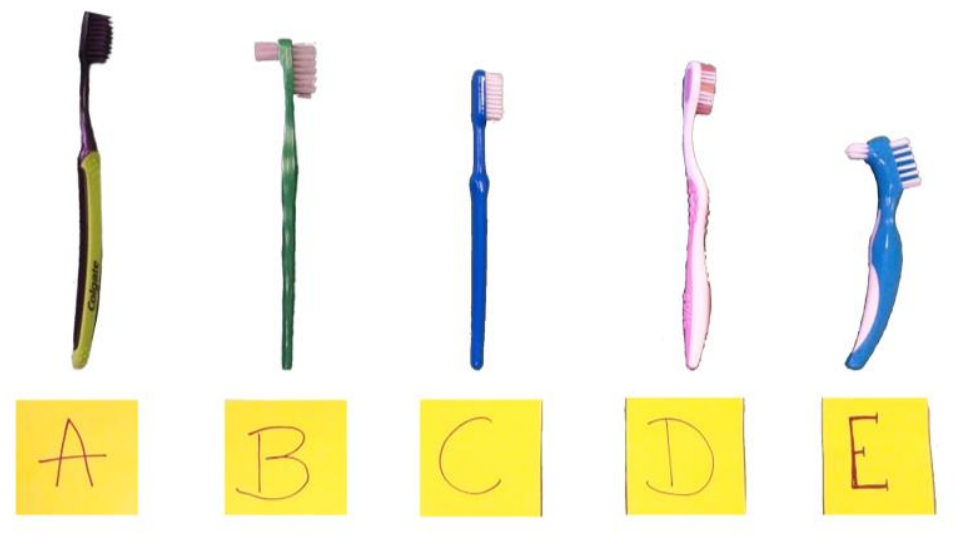

Para o presente estudo, portanto, foram considerados apenas o desempenho dos produtos nas escalas de avaliação, dados que não podem ser considerados conclusivos devido ao baixo $\mathrm{N}$ de participantes - que, no entanto, permite aos avaliadores observar diferenças entre os construtos atribuídos aos produtos, sejam eles avaliativos ou descritivos (HASSENZAHL; WESSLER, 2000). Neste sentido, conforme Gkouskos, Normark e Lundgren (2014) e em acordo com Hassenzahl e Wessler (2000), o RGT:

É especialmente útil quando se considera as percepções do usuário, necessidades, crenças e atitudes no projeto de um produto, e também pode ser usado para mapear diferenças entre artefatos, bem como semelhanças e diferenças dentro de indivíduos ou grupos de participantes (GKOUSKOS; NORMARK; LUNDGREN, 2014, p. 63, tradução nossa).

Para a aplicação do método, foram separados dois grupos de alunos da Universidade Federal do Maranhão (UFMA), sendo o primeiro grupo formado por alunos do curso de Design $(\mathrm{N}=06)$ e o segundo por alunos de outros cursos $(\mathrm{N}=05)$. Para cada participante eram apresentados um conjunto de cinco escovas de dentes, em grupos de três por vez (tríades), em combinações e ordem randomizadas e pré-definidas. Os participantes deveriam criar algum vínculo entre dois dos três produtos, por meio de uma característica comum (elemento) (representada por uma palavra ou expressão curta). Conforme Fransella, Bell e Bannister (2004, p. 15, tradução nossa), "os elementos são definidos por Kelly como 'as coisas ou eventos que são abstraídos por uma construção' e são vistos como um dos 'aspectos formais de uma construção'”. Em seguida, para cada característica gerada, os pesquisadores solicitaram que os participantes propusessem um oposto (também por meio de palavra ou expressão curta), que caracterizaria 0 construto. $O$ processo era repetido até que todas as combinações das escovas tivessem sido 
apresentadas. Os participantes, então, deveriam classificar todas escovas dentro dos construtos criados, utilizando uma escala de avaliação disponível na tabela ( $g r i d$ ) fornecida (Tabela 1).

Tabela 01. Tabela (grid) aplicada no teste. (Fonte: os autores)

\begin{tabular}{|c|c|c|c|c|c|c|c|c|c|c|c|c|c|c|c|c|c|c|c|c|c|c|c|c|c|c|c|c|c|c|c|}
\hline \multirow[t]{2}{*}{ OPOSTOS } & \multicolumn{6}{|c|}{ Escova A } & \multicolumn{6}{|c|}{ Escova B } & \multicolumn{6}{|c|}{ Escova C } & \multicolumn{6}{|c|}{ Escova D } & \multicolumn{6}{|c|}{ Escova $E$} & \multirow[t]{2}{*}{ CONSTRUTOS } \\
\hline & 0 & 1 & 2 & 3 & 4 & 5 & 0 & 1 & 2 & 3 & 4 & 5 & 0 & 1 & 2 & 3 & 4 & 5 & 0 & 1 & 2 & 3 & 4 & 5 & 0 & 1 & 2 & 3 & 4 & 5 & \\
\hline & 0 & 1 & 2 & 3 & 4 & 5 & 0 & 1 & 2 & 3 & 4 & 5 & 0 & 1 & 2 & 3 & 4 & 5 & 0 & 1 & 2 & 3 & 4 & 5 & 0 & 1 & 2 & 3 & 4 & 5 & \\
\hline & 0 & 1 & 2 & 3 & 4 & 5 & 0 & 1 & 2 & 3 & 4 & 5 & 0 & 1 & 2 & 3 & 4 & 5 & 0 & 1 & 2 & 3 & 4 & 5 & 0 & 1 & 2 & 3 & 4 & 5 & \\
\hline & 0 & 1 & 2 & 3 & 4 & 5 & 0 & 1 & 2 & 3 & 4 & 5 & 0 & 1 & 2 & 3 & 4 & 5 & 0 & 1 & 2 & 3 & 4 & 5 & 0 & 1 & 2 & 3 & 4 & 5 & \\
\hline & 0 & 1 & 2 & 3 & 4 & 5 & 0 & 1 & 2 & 3 & 4 & 5 & 0 & 1 & 2 & 3 & 4 & 5 & 0 & 1 & 2 & 3 & 4 & 5 & 0 & 1 & 2 & 3 & 4 & 5 & \\
\hline & 0 & 1 & 2 & 3 & 4 & 5 & 0 & 1 & 2 & 3 & 4 & 5 & 0 & 1 & 2 & 3 & 4 & 5 & 0 & 1 & 2 & 3 & 4 & 5 & 0 & 1 & 2 & 3 & 4 & 5 & \\
\hline & 0 & 1 & 2 & 3 & 4 & 5 & 0 & 1 & 2 & 3 & 4 & 5 & 0 & 1 & 2 & 3 & 4 & 5 & 0 & 1 & 2 & 3 & 4 & 5 & 0 & 1 & 2 & 3 & 4 & 5 & \\
\hline & 0 & 1 & 2 & 3 & 4 & 5 & 0 & 1 & 2 & 3 & 4 & 5 & 0 & 1 & 2 & 3 & 4 & 5 & 0 & 1 & 2 & 3 & 4 & 5 & 0 & 1 & 2 & 3 & 4 & 5 & \\
\hline & 0 & 1 & 2 & 3 & 4 & 5 & 0 & 1 & 2 & 3 & 4 & 5 & 0 & 1 & 2 & 3 & 4 & 5 & 0 & 1 & 2 & 3 & 4 & 5 & 0 & 1 & 2 & 3 & 4 & 5 & \\
\hline & 0 & 1 & 2 & 3 & 4 & 5 & 0 & 1 & 2 & 3 & 4 & 5 & 0 & 1 & 2 & 3 & 4 & 5 & 0 & 1 & 2 & 3 & 4 & 5 & 0 & 1 & 2 & 3 & 4 & 5 & \\
\hline
\end{tabular}

Importante frisar que o presente estudo buscou observar o funcionamento do método e a maneira como os participantes criavam seus vínculos entre as escovas de dentes; e não teve caráter conclusivo em termos de classificação dos produtos (como o melhor ou pior), tampouco buscou avaliar a qualidade das escovas.

É importante destacar também que foi aplicado um pré-teste para que se propusesse um protocolo de aplicação do teste, utilizando outros produtos na análise (canetas) e, então, permitindo ao grupo de pesquisadores se familiarizar com o método. Para fins de análise, foram considerados válidos 6 testes realizados com alunos do curso de Design e 5 dos testes feitos com alunos dos outros cursos. A amostragem mostra-se suficiente, porém, para apresentar dados relevantes ao uso do RGT.

\section{RESULTADOS E DISCUSSÕES}

O primeiro dado que chama atenção é o número de construtos gerados pelos participantes 27, agrupados em 8 dimensões construtivas. O RGT permite, nesse caso, que seja observada a tabela de frequência quanto à esses construtos, o que, numa análise inicial, pode significar que tais elementos (ou propriedades) são bastante relevantes no caso dos produtos apresentados. Considerando que não foi apresentado aos participantes qual era o foco da pesquisa (permitindo, assim, uma análise mais generalista do produto) e, por motivos lógicos (higiene e disponibilidade) não foi possível permitir um uso mais fiel das escovas de dentes por parte dos participantes, a maioria dos construtos seguiu uma lógica mais voltada à percepção do uso.

Considerando a liberdade de aplicação do teste e elaboração das grades (grids) anteriormente citadas; e a partir das respostas apresentadas nessas tabelas, a equipe de análise reuniu-se para alinhar os construtos, conforme também feito por Gkouskos, Normark e Lundgren (2014). Definiu-se, a partir daí, quais eram as "dimensões construtivas" — ou dimensões de construtos, 
$17^{\circ}$ ERGODESIGN \& USIHC 2019

PUC-Rio, 11 a 13 de dezembro

Rio de Janeiro, RJ, Brasil $17^{\circ}$ Ergodesign - Congresso Internacional de Ergonomia e Usabilidade de Interfaces Humano Tecnológica: Produto, Informações Ambientes Construídos e Transporte

$17^{\circ}$ USIHC - Congresso Internacional de Ergonomia e Usabilidade de Interfaces Humano Computador

considerando que não foi encontrada uma tradução padrão quanto ao termo "Construct Dimensions", utilizado por Gkouskos, Normark e Lundgren (2014). Em resumo, os elementos apontados pelos participantes junto de seus opostos foram chamados "construtos" (FALLMAN, 2006; GKOUSKOS; NORMARK, 2012; HOGAN; HORNEKER, 2013), e tais construtos foram mesclados por similaridade, conforme discussão da equipe pesquisadora, de maneira a gerar as "dimensões construtivas".

A dimensão construtiva que mais aparece nas respostas é utilização da escova (27 menções, $25 \%$ do total), enquanto o construto mais mencionado refere-se ao formato da escova/curvatura (14 menções, $12 \%$ do total), fazendo parte de outra dimensão construtiva: formato. Entre os alunos do curso de Design, os construtos localização das cerdas, pega e uso de cores mostraram-se bastante relevantes - $10 \%$ das menções do grupo cada - , diferentemente dos participantes dos demais cursos, que mencionaram mais o construto formato da escova/curvatura (18\% do total de construtos). Já quando analisadas, as dimensões construtivas, ambos os grupos apresentaram comportamentos semelhantes, com maior número de menções para a dimensão utilização da escova: $24 \%$ entre os alunos de outros cursos e $27 \%$ entre os alunos de design.

Chama atenção, também, o fato de 3 dimensões serem avaliativas (32\% dos construtos estavam nestas dimensões para alunos de design, 28\% para alunos de outros cursos) e 5 serem descritivas (com 68\% dos construtos nestas dimensões para alunos de design, $72 \%$ para os de outros cursos). É possível, ainda, separar os construtos por classificações Avaliativas ou Descritivas (HASSENZAHL; WESSLER, 2000; GKOUSKOS; NORMARK; LUNDGREN, 2014) — no estudo apresentado, 14 construtos são totalmente descritivos, 9 são avaliativos e 4 ficaram como "predominantemente descritivos", já que não foi possível chegar a uma conclusão e agrupá-los nas outras categorias (Tabela 02).

Tabela 02. Classificação das dimensões de construtos nas categorias "descritiva" e "avaliativa". (Fonte: os autores)

\begin{tabular}{|c|c|c|}
\hline Dimensão construtiva & Construtos & Categoria \\
\hline Materiais & $\begin{array}{c}\text { Diversidade de materiais, } \\
\text { peso, apelo ecológico. }\end{array}$ & Descritiva \\
\hline Estética & $\begin{array}{c}\text { Uso de cores, destaque para } \\
\text { a marca, complexidade do } \\
\text { produto/projeto, apelo } \\
\text { estético. }\end{array}$ & $\begin{array}{c}\text { Predominantemente } \\
\text { Descritiva }\end{array}$ \\
\hline Cerdas & $\begin{array}{c}\text { Altura das cerdas, formato } \\
\text { das cerdas, firmeza das } \\
\text { cerdas, localização das } \\
\text { cerdas, } \\
\text { densidade/quantidade de } \\
\text { cerdas. }\end{array}$ & Descritiva \\
\hline Dimensionamento & $\begin{array}{c}\text { Tamanho da cabeça, largura } \\
\text { da escova, tamanho, } \\
\text { tamanho do cabo. }\end{array}$ & Descritiva \\
\hline
\end{tabular}




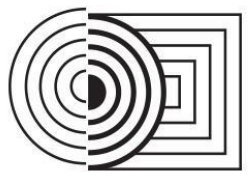

$17^{\circ}$ ERGODESIGN \& USIHC 2019

PUC-Rio, 11 a 13 de dezembro

Rio de Janeiro, RJ, Brasil $17^{\circ}$ Ergodesign - Congresso Internacional de Ergonomia e Usabilidade de Interfaces Humano Tecnológica: Produto, Informações Ambientes Construídos e Transporte

$17^{\circ}$ USIHC - Congresso Internacional de Ergonomia e Usabilidade de Interfaces Humano Computador

\begin{tabular}{|c|c|c|}
\hline Formato & $\begin{array}{c}\text { Formato da cabeça, } \\
\text { formato/curvatura da escova. }\end{array}$ & Descritiva \\
\hline Resistência & $\begin{array}{c}\text { Grau de flexibilidade da } \\
\text { escova, resistência. }\end{array}$ & Avaliativa \\
\hline Utilização da escova & $\begin{array}{c}\text { Facilidade de uso, } \\
\text { grip/aderência do cabo, pega, } \\
\text { multifuncionalidade, } \\
\text { estabilidade. }\end{array}$ & Avaliativa \\
\hline Portabilidade & $\begin{array}{c}\text { Facilidade de transporte, } \\
\text { facilidade de } \\
\text { armazenamento. }\end{array}$ & Avaliativa \\
\hline
\end{tabular}

Dentro das dimensões avaliativas ainda foram encontradas peculiaridades, como o fato de alguns construtos terem sido elaborados a partir de propriedades consideradas "positivas", ao passo que outros foram baseados em propriedades "negativas". Um participante, por exemplo, gerou em uma mesma dimensão (grip/aderência) propriedades consideradas positivas ("material do cabo facilita a escovação" e "o design melhora o ato de segurar a escova") e uma considerada negativa ("o emborrachamento dificulta o deslize da escova"). Dessa forma, para uma análise mais precisa foi necessário gerar um score para as dimensões isoladamente.

Assim foi possível outra análise, quanto ao desempenho de cada produto. Considerando apenas as dimensões construtivas, foi possível posicionar cada uma das 5 escovas em espectros gráficos. Importante frisar, porém, que não necessariamente o fato de um produto obter pontuação máxima na escala de avaliação significa ser bom ou ruim (nos construtos descritivos), porém nos construtos interpretados como avaliativos é possível inferir se um produto possui melhor ou pior aceitação entre os participantes do que o outro (Tabela 03).

Tabela 03. Pontuação total "positiva" e "negativa", considerando apenas as dimensões construtivas avaliativas.

(Fonte: os autores)

\begin{tabular}{|c|c|c|c|}
\hline Escova & Pontuação positiva & Pontuação negativa & Participantes \\
\hline A & 48 & 11 & Curso de design \\
\hline B & 37 & 5 & Curso de design \\
\hline C & 27 & 8 & Curso de design \\
\hline D & 55 & 15 & Curso de design \\
\hline E & 47 & 8 & Curso de design \\
\hline A & 36 & 5 & Outros cursos \\
\hline B & 17 & 0 & Outros cursos \\
\hline
\end{tabular}




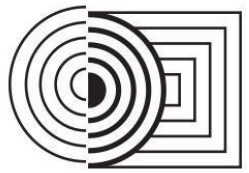

$17^{\circ}$ ERGODESIGN \& USIHC 2019

PUC-Rio, 11 a 13 de dezembro

Rio de Janeiro, RJ, Brasil

$17^{\circ}$ Ergodesign - Congresso Internacional de Ergonomia e Usabilidade de Interfaces Humano Tecnológica: Produto, Informações Ambientes Construídos e Transporte

$17^{\circ}$ USIHC - Congresso Internacional de Ergonomia e Usabilidade de Interfaces Humano Computador

\begin{tabular}{|c|l|l|l|}
\hline C & 24 & 0 & Outros cursos \\
\hline D & 44 & 1 & Outros cursos \\
\hline E & 22 & 0 & Outros cursos \\
\hline
\end{tabular}

Conforme observado na Tabela 03, nota-se por exemplo que a escova $D$ teve maior aceitação nos dois grupos de participantes. Enquanto isso, as escovas com maior rejeição variam - no grupo de participantes do curso de design, a escova mais rejeitada é a $\mathrm{C}$, enquanto no grupo de participantes de outros cursos é a escova B.

Conforme também apresentado por Fernandes (2005), o método RGT possui algumas limitações que se mostraram evidentes na condução do estudo com as escovas de dentes. Além do já citado problema com o tempo de execução dos testes, outras dificuldades apareceram durante a pesquisa. Corroborando com o exposto pelo autor, neste estudo também observou-se problemas quanto à expertise dos entrevistadores (todos ainda estavam em fase de familiarização com o método), de entendimento dos conceitos e, principalmente, da dificuldade dos participantes que, por entender que as escovas de dentes eram produtos muito simples do ponto de vista construtivo e conceitual, em algum momento tiveram bastante dificuldade para elaborar construtos.

Também apresentado por Fernandes (2005), outro problema encontrado foi relacionado à tabulação e interpretação dos dados. Em que pese a obra apresentada por Fransella, Bell e Bannister (2004), na qual explicam de forma detalhada o funcionamento do RGT, a falta de conhecimento prático por parte da equipe pesquisadora pode se tornar um grande impeditivo para a correta utilização das informações obtidas.

\section{CONCLUSÕES}

Apesar da evidente complexidade, o método RGT para a avaliação de produtos se mostra uma ferramenta com um expressivo potencial, permitindo à designers e desenvolvedores obter dados de extrema importância, tanto para novos projetos quanto para re-designs. Conforme observado no teste das escovas de dentes, a ótica do potencial usuário dos produtos é um importante aliado do projeto, principalmente levando-se em consideração princípios de design centrado no usuário, assim como outras vertentes da ergonomia e áreas correlatas.

O RGT pode ser aplicado em diversas fases do projeto, sempre buscando obter informações que auxiliem o processo de desenvolvimento do produto. Aplicado com parcimônia pode trazer resultados que outros métodos tradicionais não conseguem detectar. A versatilidade da obtenção de dados qualitativos e quantitativos ainda permite uma série de interpretações, passíveis de discussões entre equipes e com os próprios usuários (utilizando outras técnicas em conjunto, como entrevistas ou grupos focais), e mesmo que o teste não traga resultados conclusivos ou evidentes, ainda permite que o designer saiba que rumo tomar na condução de novas abordagens durante o desenvolvimento do projeto.

É recomendado, porém, a familiarização com os procedimentos, o protocolo do estudo e principalmente a expertise para a tabulação, análise e interpretação dos dados se mostra um grande desafio para a equipe. 


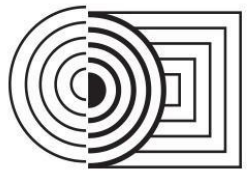

\section{$17^{\circ}$ ERGODESIGN \& USIHC 2019 \\ PUC-Rio, 11 a 13 de dezembro \\ Rio de Janeiro, RJ, Brasil}

$17^{\circ}$ Ergodesign - Congresso Internacional de Ergonomia e Usabilidade de Interfaces Humano Tecnológica: Produto, Informações Ambientes Construídos e Transporte

$17^{\circ}$ USIHC - Congresso Internacional de Ergonomia e Usabilidade de

Interfaces Humano Computador

Por fim, este estudo propõe, como desdobramento, nova aplicação do RGT com outros produtos e interfaces, utilizando um $\mathrm{N}$ mais expressivo. Tais alterações podem enriquecer ainda a análise dos dados, ao possibilitar a extração de mais informações do teste, resultando em melhor entendimento do método para futuras pesquisas e aplicações no campo de design.

\section{REFERÊNCIAS BIBLIOGRÁFICAS}

FALLMAN, D. Integrating User Experience into the Design Process with the Repertory Grid Technique: Some Preliminary Notes. WP9 Workshop on Innovative Approaches for Evaluating Affective Systems, Stockholm, Sweden, Jan 9-11, 2006.

FERNANDES, Eugénia M.; ALMEIDA Leandro S., ed. lit. Métodos e técnicas de avaliação: contributos para a prática e investigação psicológicas. Braga: Universidade do Minho. Centro de Estudos em Educação e Psicologia, 2001. ISBN 972-8098-98-7. p. 77-107.

FRANSELLA, F.; BELL, R.; BANNISTER, D. A Manual for Repertory Grid Technique. Londres: Academic Press, 2003. ISBN 0470854901.

GKOUSKOS, D.; NORMARK, C. J. What Drivers Really Want: Investigating Dimensions in Automobile User Needs. in International Journal of Design, v. 8, n. 1, p. 59-71, 2014.

GKOUSKOS, D.; NORMARK, C. J. Exploring User Needs in Automobiles. International Design Conference, Croatia, p. 1369-1376, 2012.

HASSENZAHL, M.; WESSLER, R. Capturing Design Space From a User Perspective: The Repertory Grid Technique Revisited. in International Journal of Human-Computer Interaction. Lawrence Erlbaum Associates, 2000. DOI 0.1207/S15327590IJHC1203\&4_13, v. 12, n. 3-4, p. 441-459.

HEINE, K. Using Personal and Online Repertory Grid Methods for the Development of a Luxury Brand Personality. The Electronic Journal of Business Research Methods. Academic Conferences, 2009. v. 7, n. 1, p.25-38.

HERNANDEZ, José M. A utilização do RGT (Repertory Grid Technique) na mensuração de imagem de shopping centers. RAl - Revista de Administração e Inovação, São Paulo, v. 2, n. 2, p. 19-32, 2005.

HOGAN, T.; HORNECKER, E. Blending the repertory grid technique with focus groups to reveal rich design relevant insight. UK, New Castle, 2013.

KARAPANOS, E.; MARTENS, J-B. The quantitative side of the Repertory Grid Technique: some concerns. Cornell University: Human-Computer Interaction. Florence, 2008.

TOMICO, O. et al. The Repertory Grid Technique as a Method for the Study of Cultural Differences. in International Journal of Design, v. 3, n. 3, p. 55-63, 2009.

\section{Agradecimentos}

O presente trabalho é parte das atividades do Projeto DINTER UFMA/UNESP e foi desenvolvido com apoio da Coordenação de Aperfeiçoamento de pessoal de Nível Superior - Brasil (CAPES); da Fundação de Amparo à Pesquisa e ao Desenvolvimento Científico e Tecnológico do Maranhão (FAPEMA - edital 012/2016, processo 04206/2016) e do CNPq (Processo 304619/2018-3). Agradecemos também à equipe do Fabrique Design - Núcleo de Prototipagem UFMA do CCET Centro de Ciências Exatas e Tecnologia da Universidade Federal do Maranhão. 